\title{
СРАВНИТЕЛЬНАЯ КОНКУРЕНТОСПОСОБНОСТЬ КАК ВАЖНЕЙШИЙ ИНСТРУМЕНТ ЭФФЕКТИВНОГО И УСТОЙЧИВОГО РАЗВИТИЯ ПРОМЫШЛЕННЫХ ПРЕДПРИЯТИЙ
}

\section{COMPARATIVE COMPETITIVENESS AS THE MOST IMPORTANT TOOL FOR EFFECTIVE AND SUSTAINABLE DEVELOPMENT OF INDUSTRIAL ENTERPRISES}

\section{Starkova}

Summary: The purpose of this article is to study the relationship and role of competitive advantages and strategies for their formation as the most important tools in improving the competitiveness of an industrial enterprise, ensuring its sustainable and effective development.

Using the necessary tools, including the methods of logical, comparative and economic-statistical analysis, the article proposes a scheme and mechanism for the interconnection of factors, strategies for the formation of competitive advantages and measures for their implementation in an industrial enterprise with an indication of market niches.

This article proposes three strategies for the formation of competitive advantages - leadership in price, product quality, differentiation of consumers and their relationship with external (value for the consumer) and internal (value for the manufacturer) competitive advantage.

It is concluded that the production of competitive products, sustainable and efficient development of an industrial enterprise is possible only with the interconnection of factors to increase competitiveness, strategies for creating competitive advantages, measures to implement a strategy and evaluate the competitiveness of products and the enterprise itself.

Keywords: competitiveness, competitive advantages, competitive strategies, market, consumers, enterprise, price, costs.

\author{
Старкова Мария Михайловна \\ К.э.н., доцент, Московский авиационный институт \\ (национальный исследовательский университет) \\ starkova_maria@mail.ru
}

Аннотация: Цель данной статьи состоит в исследовании взаимосвязи и роли конкурентных преимуществ и стратегий их формирования как важнейших инструментов в повышении конкурентоспособности промышленного предприятия, обеспечении его устойчивого и эффективного развития.

В статье с помощью необходимого инструментария, включающего методы логического, сравнительного и экономико-статистического анализа предложена схема и механизм взаимосвязи факторов, стратегий формирования конкурентных преимуществ и мероприятий по их реализации на промышленном предприятии с указанием рыночных ниш.

В настоящей статье предложены три стратегии формирования конкурентных преимуществ - лидерство в цене, качество продукции, дифференциация потребителей и отражена их связь с внешним (ценность для потребителя) и внутренним (ценность для производителя) конкурентным преимуществом. Сделан вывод о том, что производство конкурентоспособной продукции, устойчивое и эффективное развитие промышленного предприятия возможно только при взаимосвязи факторов повышения конкурентоспособности, стратегий формирования конкурентных преимуществ, мероприятий по реализации стратегии и оценки конкурентоспособности продукции и самого предприятия.

Ключевые слова: конкурентоспособность, конкурентные преимущества, конкурентные стратегии, рынок, потребители, предприятие, цена, издержки.

- конкурентоспособность продукции (индивидуальный уровень);

- конкурентоспособность предприятия (микроуровень);

- конкурентоспособность отрасли (мезоуровень);

- конкурентоспособность региона (мезоуровень)

- конкурентоспособность национальной экономики в целом (макроуровень).

Независимо от уровня управления сущность конкурентоспособности в целом одинакова - это способность страны, региона, отрасли, предприятия опережать конкурентов в достижении поставленных целей, т.е. направленность на получение конкурентных преимуществ для укрепления своих позиций на рынке и получения на основе этого прибыли. Все рассматриваемые уровни конкурентоспособности взаимосвязаны, дополняют, 


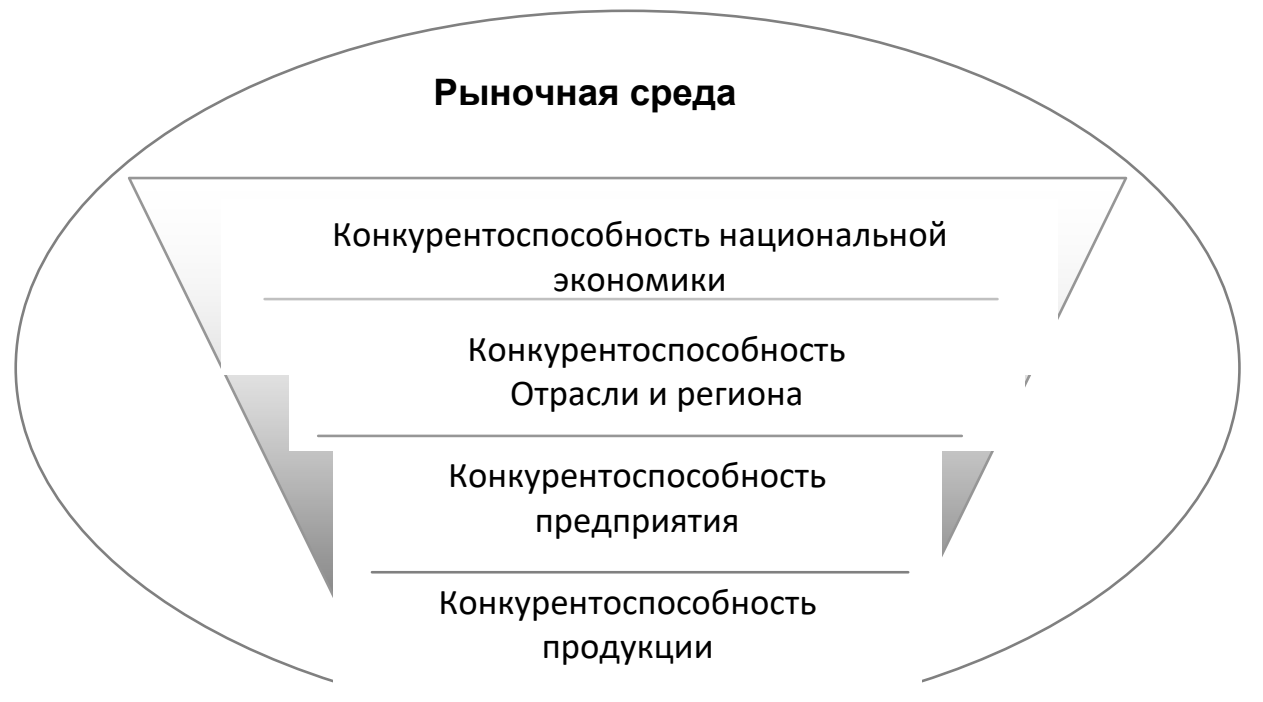

Рис. 1. Конкурентоспособность на различных уровнях управления

обеспечивают и определяют друг друга и в наибольшей степени это касается взаимосвязи между категориями «конкурентоспособность предприятия» и «конкурентоспособность продукции».

Имеется в виду тот факт, что конкурентные преимущества и конкурентные стратегии разрабатываются в отношении продукции, но направлены в целом на повышение конкурентоспособности промышленного предприятия.

Конкурентные преимущества предприятия определяются внешними, проявление которых в малой степени зависит от предприятия, и внутренними факторами, почти целиком определяемыми руководством предприятия, причем определяющими являются внешние факторы. В таблице 1 приведен перечень внешних факторов конкурентного преимущества промышленного предприятия.

Конкурентные преимущества, полученные на основе использования и применения факторов, являются наиболее значимыми и основными определяющими инструментами сравнительной конкурентоспособности промышленных предприятий, обеспечивающими успех в конкурентной борьбе.

Позицию предприятия в конкретной отрасли промышленности, как известно, определяет именно конкурентное преимущество, которое существует тогда, когда предприятие может получить рентабельность выше средней в данной отрасли или сегменте рынка $[11 ; 12]$. Достигнуть же такую рентабельность позволяют именно конкурентные преимущества, а именно осязаемые и не-

Перечень внешних факторов конкурентного преимущества промышленного предприятия

\begin{tabular}{|l|l|}
\hline \multicolumn{1}{|c|}{ Внешний фактор конкурентного преимущества } & \multicolumn{1}{|c|}{\begin{tabular}{c}
\multicolumn{1}{c|}{ Мероприятия для достижения и использования конкурентного } \\
преимущества
\end{tabular}} \\
\hline \multicolumn{1}{|c|}{1} & \multicolumn{1}{c|}{\begin{tabular}{c}
\multicolumn{1}{|c|}{} \\
\hline Уровень конкурентоспособности предприятия
\end{tabular}} \\
\hline $\begin{array}{l}\text { Правовое регулирование функционирования экономики страны и } \\
\text { регионов (отрасли, города) }\end{array}$ & $\begin{array}{l}\text { Переработка законодательной базы функционирования экономики как системы } \\
\text { кодексов и прав }\end{array}$ \\
\hline $\begin{array}{l}\text { Научный уровень управления экономикой страны, отрасли и } \\
\text { регионов }\end{array}$ & $\begin{array}{l}\text { Применение экономических законов функционирования рыночных отношений, } \\
\text { законов организации, научный подход к управлению на всех уровнях }\end{array}$ \\
\hline Национальная система стандартизации и сертификации & $\begin{array}{l}\text { Активизация работ в этой области, усиление контроля над соблюдением } \\
\text { международных стандартов и соглашений }\end{array}$ \\
\hline $\begin{array}{l}\text { Качество информационного обеспечения управления на всех } \\
\text { уровнях }\end{array}$ & $\begin{array}{l}\text { Создание единых национальных центров по отраслям народного хозяйства, } \\
\text { отвечающих современному состоянию науки и техники }\end{array}$ \\
\hline Налоговые ставки в стране и регионе & Пересмотр налоговой системы, состыковка и унифицирование ставок \\
\hline Процентные ставки в стране и регионе & $\begin{array}{l}\text { Пересмотр системы процентных ставок на всех уровнях управления и } \\
\text { направлениях инвестирования }\end{array}$ \\
\hline
\end{tabular}


осязаемые активы, которыми владеет предприятие, те сферы деятельности, которые стратегически важны для данного бизнеса, и которые позволяют предприятию побеждать в конкурентной борьбе.

Следовательно, можно сказать, что конкурентное преимущество промышленного предприятия [11;12] проявляется как преобладание над конкурентами на рынке промышленной продукции (или его сегменте) и характеризуется как «продуктивность использования ресурсов», критерием которой выступает показатель рентабельности производства.

Конкурентное преимущество, которым обладают на конкретных рынках различные соперники (конкуренты), является существенным фактором климата, или конкурентной ситуации, на рынке производимой промышленной продукции. Конкурентное преимущество, которое создает предприятию определенное превосходство над своими прямыми конкурентами, определяется набором характеристик, свойств продукции и оценивается относительным, сравнительным состоянием, положением предприятия по отношению к конкуренту, занимающему наилучшую позицию на рынке данной промышленной продукции или в сегменте рынка.

Конкурентные преимущества, зависящие от факторов развития производства, являются производными для выработки стратегии конкурентных преимуществ, которые в свою очередь, могут быть внешними и внутренними.

Конкурентное преимущество является внешним, если оно основано как на отличительных качествах промышленной продукции, образующих «ценность для потребителя», так и ввиду сокращения издержек или повышения эффективности. Внешнее конкурентное преимущество увеличивает рыночную силу промышленного предприятия, т.е. способность предприятия заставить рынок принять цену продукции, более высокую, чем у приоритетных (самых опасных) конкурентов, но не обеспечивающих соответствующего отличительного качества $[3 ; 6]$.

Внутреннее конкурентное преимущество базируется на первенстве предприятия в отношении издержек производства, управления предприятием или конкретной продукцией, которые создают «ценность для производителя» и меньшую себестоимость, чем у конкурента. Это преимущество может создаваться благодаря проведению стратегии доминирования по издержкам за счет внедрения организационного и производственного нововведения на предприятии [3;6].

Таким образом, соотношение «рыночной силы» и «производительности» может характеризовать уровень конкурентного превосходства предприятия перед кон- курентами. Исходя из нацеленности промышленного предприятия на достижение внешних или внутренних конкурентных преимуществ, на нем разрабатываются соответствующие стратегии достижения конкурентного преимущества.

Возможность формирования конкурентных преимуществ зависит от ряда факторов, однако при выборе конкурентной стратегии внешнего контура, прежде всего, следует ориентироваться на запросы потребителей и убедиться в том, что достигнутые конкурентные преимущества воспринимаются потребителями; при выборе стратегии внутреннего конкурентного преимущества ориентация, прежде всего, - на интересы самого промышленного предприятия - производителя продукции.

Следует отметить, что первая конкурентная стратегия промышленного предприятия - это лидерство в цене, при которой центром внимания предприятия, при разработке и производстве продукции, становятся издержки, являющиеся важнейшей компонентой при формировании стратегии ценообразования на промышленную продукцию.

Данная стратегия, прежде всего, соответствует внутренней конкурентной стратегии и представляет ценность для производителя (промышленного предприятия) и направлена на снижение издержек и цены продукции.

Ценовые решения неизбежно привязаны к решениям об объемах реализации, которые связаны с производственными, маркетинговыми и управленческими издержками. Имеется в виду, что в дополнение к эффективности внутренних издержек предприятие также может снизить и внешние издержки за счет правильного выбора и управления своими контактами с потребителями и поставщиками $[9,59-68]$.

Во-первых, за счет получения дополнительной экономии от концентрации на покупателе путем сосредоточения маркетинговых исследований на одном или двух рыночных сегментах; при этом значимый элемент экономии состоит в издержках на продвижение и на рекламную деятельность.

Во-вторых, за счет экономии от интеграции материально-технического обеспечения (проведение логистических мероприятий). Улучшение взаимодействия с поставщиками может минимизировать стоимость товарно-материальных запасов, а более высокая координация в ценообразовании сделать промышленное предприятие и его поставщиков более конкурентоспособными и рентабельными. Экономия может быть достигнута и на другом конце логистической цепочки - в процессе реализации продукции за счет качественного сервисного 
послепродажного обслуживания. Эффективность реализации может значительно повлиять на общее ценовое преимущество предприятия.

В-третьих, особое значение в российских условиях управления издержками имеет их воздействие на цену. Учитывая, что система рыночных отношений предполагает управление не только себестоимостью, но и всеми затратами предприятия, то и механизм влияния издержек на цены должен учитывать всю совокупность затрат и эту функцию призваны выполнять центры управления затратами [9,59-58].

Минусом стратегии ценообразования является то, что данный тип преимуществ зависит от существования особых условий рынка и эффективен не во всех отраслях промышленности. Так, существенную роль играет уровень стандартизации продукции, рыночной власти потребителей и эластичности спроса по цене, доступности источников дешевых ресурсов. При этом существует риск потери конкурентного преимущества вследствие появления новой продукции или технологий.

Для позитивного развития данной стратегии предлагается использование дифференцированного (кастомизированного) ценообразования, в также более жесткая увязка цен с реальными уровнями издержек.

Качество промышленной продукции является второй стратегией и соответствует внешним конкурентным преимуществам, отражая ценность для потребителя. При данной стратегии промышленное предприятие намерено придать продукции отличительные и необходимые свойства, те, что должны соответствовать потребностям потребителя продукции и которые он готов оплатить. Стратегия качества направлена на то, чтобы сделать продукцию отличительной от той, какой ее делают предприятия-конкуренты. При этом целевая направленность промышленного предприятия состоит не в том, чтобы увеличить ценовую составляющую, т.к. дополнительную прибыль оно может получить и за счет увеличения объемов реализации продукции, и за счет стабилизации потребления вне зависимости от колебаний спроса на рынке. Поэтому предприятие стремится сохранить издержки на прежнем уровне, для стабилизации цены.

Для реализации стратегии качества создания конкурентных преимуществ значимым является концентрация внимания на потребительских приоритетах и интересах покупателя и поэтому для предприятия важно, чтобы необычность продукта, его новизна или уникальность имели ценность для покупателя. С учетом этих аспектов стратегия качества предполагает в качестве исходной точки изучение интересов потребителя и в этом стыкуется с третьей стратегией - дифференциацией покупате- лей. Для досконального знания интересов потребителей промышленному предприятию необходимо:

- изучать потребности и потребительские критерии, лежащие в основе приобретения той или иной промышленной продукции (цена, функциональные свойства, условия производства, гарантии, срок поставки и т.п.);

- выявлять и определять факторы, формирующие представление покупателя о промышленной продукции (источники информации о свойствах продукта).

- успешно реализованная стратегия качества позволит промышленному предприятию:

- устанавливать премиальную наценку на свою продукцию;

- реализовывать больший объем продукции (за счет дополнительного числа потребителей);

- создать привлекательный имидж предприятия среди покупателей промышленной продукции.

Такое конкурентное преимущество является достаточно устойчивым, так как качественные признаки не могут быть имитированы конкурентами оперативно и без привлечения значительных затрат. Однако трудность достижения преимущества за счет качества обуславливается проблемой поиска и выделения в продукции тех характеристик, которые представляют для потребителей особую ценность. Существует риск неправильного отбора, когда рост цены не будет соответствовать новому качеству продукции.

К другим рискам можно отнести следующие: предприятие не осознает значимость ценности продукции для потребителей; копирование продукции конкурентами может нивелировать появившуюся качественную разницу в продукции; предприятие не осуществляет никаких действий по доведению до потребителей информации о ценности продукции.

Третьей стратегией, которую промышленное предприятие может использовать для создания конкурентных преимуществ своей продукции, является дифференциация потребителей, т.е. имеется в виду учет интересов конкретных потребителей. В этом случае предприятие разрабатывает и реализует свою продукцию для нужд конкретных потребителей. Целенаправленное создание промышленного продукции предполагает, что удовлетворяется потребность определенной группы потребителей промышленной продукции либо же создается специфическая система доступа к продукции (система продажи и доставки продукта). Данная стратегия предполагает проведение аналогичных мероприятий, предлагаемых для стратегии качества по изучению предпочтений потребителей, но с расширением их спектра за счет выделения и формирования групп потребителей. 
Для реализации этой задачи промышленным предприятиям предлагается осуществлять как макросегментацию, так и микросегментацию рынка продукции. Имеется в виду разделение рынка производимой продукции в два этапа, соответствующих двум различным уровням разделения рынка. На первом этапе производится макросегментация, состоящая в идентификации «рынка промышленной продукции», тогда как на втором этапе осуществляется микросегментация, предполагающая выявление внутри каждого идентифицированного рынка «сегментов» (групп) конкретных потребителей.

Кроме названных стратегий конкурентных преимуществ необходимо выделить возможность формирования конкурентных преимуществ за счет интеграции конкурентных стратегий. Например, в случае интеграции стратегии «конкурентоспособные цены» и стратегии качества промышленное предприятие может обеспе- чивать производство продукции с максимальным качеством при наименьших издержках.

При включении дополнительно к этим двум стратегиям третьей стратегии конкурентных преимуществ промышленное предприятие, концентрируясь на низких издержках, должно обеспечить качество продукции необходимое для конкретной группы потребителей.

Резюмируя, заметим, что успешное производство конкурентоспособной продукции, а также эффективное и устойчивое развитие промышленного предприятия возможно только при оптимальном сочетании и взаимосвязи факторов повышения конкурентоспособности, разработки стратегии формирования конкурентных преимуществ, выработки мероприятий по реализации стратегии и эффективной оценки конкурентоспособности продукции и самого предприятия.

\section{ЛИТЕРАТУРА}

1. Азоев Г.Л. Конкуренция: анализ, стратегия и практика. - М.: Центр экономики и маркетинга, 2012. - 356 с.

2. Адрианов В.Н. Конкурентоспособность России в мировой экономике. //МЭ и МО. - 2006. -№3.

3. Головачев А.С. Конкурентоспособность организации: учеб.пособие / А.С. Головачев. - Минск: Выш. шк., 2012. - 319 с

4. Горбашко Е.А., Коровкин И.А. Конкурентоспособность продукции и ее обеспечение в условиях экономики переходного периода // Партнеры и конкуренты. - 2000. - № 9. - .

5. Глухов А.Н. Информационные аспекты обеспечения конкурентоспособности промышленных товаров: Автореф. дисс. на соиск. учен. степ. канд. экон. наук: (08.00.05) / [Моск. гос. технол. ун-т «Станкин»]. - М., 2000.

6. Долинская М.Г., Соловьев И.Н. Маркетинг и конкурентоспособность промышленной продукции. М.: Финансы и статистика, 2011.

7. Калюжнова Н.Я. Конкурентоспособность регионов: теория и методология анализа в контексте современного экономического развития. Дисс. на соискание ученой степени д.э.н. ИГУ, 2004.

8. Квасникова В.В., Жучкевич О.Н. Конкурентоспособность товаров и организаций. Практикум: Учебное пособие / В.В. Квасникова, О.Н. Жучкевич. - М.: НИЦ ИНФРА-М; Мн.: Нов. знание, 2013. - 184 с.

9. Кротков А.М., Еленева Ю.Я. Конкурентоспособность предприятий: подходы к обеспечению, критерии, методы оценки // Маркетинг в России и за рубежом. - 2001. - № 6. -

10. Маркова В.Д. Стратегический менеджмент. - Новосибирск, Инфра-М, Сибирское отделение, 2005. -200с.

11. Портер М. Конкурентная стратегия: методика анализа отраслей и конкурентов. М.: Альпина Паблишер, 2011. -453с.

12. Портер М. Конкуренция = On Comhttition: Пер. с англ./Под ред. Я.В. Заблоцкого. М.: Издательский дом «Вильямс». 2010. - 610 с.

13. Старкова М.М. Сравнительная оценка конкурентоспособности промышленного предприятия//Экономический журнал Экономика: вчера, сегодня, завтра, том 8, №9А, 2018.Изд-во «Аналитика Родис».

14. Фатхутдинов Р.А. Конкурентоспособность организации в условиях кризиса: экономика, маркетинг, менеджмент. - М., 2002.

15. Фатхутдинов Р.А. Управление конкурентоспособности организации: учеб. пособие / Р.А. Фатхутдинов. - М.: Изд-во ЭКСМО, 2012. - 544 с.

16. Щербакова Л.В., Тугушева В.Р. - Современные подходы к оценке конкурентоспособности предприятия // Известия ПГПУ им. В.Г. Белинского. - 2012. - № 28.

(c) Старкова Мария Михайловна (starkova_maria@mail.ru).

Журнал «Современная наука: актуальные проблемы теории и практики» 